\title{
ANISOTROPY OF SUSCEPTIBILITY AND OPTICAL INVESTIGATION OF THE ANTIFERROMAGNETIC PHASE TRANSITION IN $\mathrm{U}_{1-x} \mathrm{Ce}_{x} \mathrm{Ru}_{2} \mathrm{Si}_{2}$
}

\author{
M. Mihalik, S. MaT̃aš, D. Macko, M. Timko \\ Institute of Experimental Physics, Slovak Academy of Sciences \\ Watsonova 47, 04353 Košice, Slovak Republic \\ AND A.A. Menovsky \\ Van der Waals-Zeeman Institute, University of Amsterdam \\ Valckenierstraat 67, 1018 XE Amsterdam, The Netherlands
}

Temperature dependence of magnetic susceptibility of $\mathrm{U}_{1-x} \mathrm{Ce}_{x} \mathrm{Ru}_{2} \mathrm{Si}_{2}$ single crystals $(x=0,0.025,0.05,0.075)$ has been studied at temperatures from $4.2 \mathrm{~K}$ up to room temperature and in magnetic fields applied along and perpendicular to the $c$-axis. The attention was paid to anomalies connected with the antiferromagnetic phase transition, which are present in crystals with $x \leq 0.05$. Far-infrared measurements of $\mathrm{U}_{0.99} \mathrm{Ce}_{0.01} \mathrm{Ru}_{2} \mathrm{Si}_{2}$ single crystal have been studied for the first time. The measurements support the assumption that the origin of magnetic ordering in $\mathrm{U}_{0.99} \mathrm{Ce}_{0.01} \mathrm{Ru}_{2} \mathrm{Si}_{2}$ is the same as in heavy-fermion superconductor $\mathrm{URu}_{2} \mathrm{Si}_{2}$ and can be interpreted as being due to a spin-density-wave gap.

PACS numbers: 72.15.-v, 75.20.Hr, 75.30.Hb

$\mathrm{URu}_{2} \mathrm{Si}_{2}$ is the first heavy-fermion system in which an unusual coexistence of superconductivity and magnetism has been discovered. It exhibits a superconducting transition at $T_{\mathrm{c}}=1.2 \mathrm{~K}$ and an antiferromagnetic transition at around $T_{\mathrm{N}}=17.5 \mathrm{~K}$. The latter transition is associated with the formation of weak antiferromagnetic ordering [1-3]. The nature of the magnetic order is still an open question, since no model can explain the extremely small magnetic moment of an order of $\approx 0.04 \mu_{\mathrm{B}}$. On the other hand, even the type of nontrivial pairing ( $d$ - or $p$-wave) with non-zero orbital momentum in the superconducting phase is still uncertain. It is believed that the interaction between the superconducting and magnetic order parameters can lead to an additional symmetry breakdown and further transitions inside the superconducting state in unconventional superconductors. 
In this paper we pay attention to the antiferromagnetic phase transition in $\mathrm{U}_{1-x} \mathrm{Ce}_{x} \mathrm{Ru}_{2} \mathrm{Si}_{2}$ and to magnetic properties of this heavy-fermion system in a paramagnetic state. The temperature dependence of magnetic susceptibility of $\mathrm{U}_{1-x} \mathrm{Ce}_{x} \mathrm{Ru}_{2} \mathrm{Si}_{2}$ single crystals $(x=0,0.025,0.05,0.075)$ has been studied at temperatures from $4.2 \mathrm{~K}$ to room temperature and in magnetic fields applied along and perpendicular to the $c$-axis. In this paper we extend our previous study of $\mathrm{U}_{1-x} \mathrm{Ce}_{x} \mathrm{Ru}_{2} \mathrm{Si}_{2}$ system [4-6] to new and better single crystals. A special attention was paid to study magnetic susceptibility in magnetic fields applied perpendicular to the $c$-axis. Far-infrared properties of $\mathrm{U}_{0.99} \mathrm{Ce}_{0.01} \mathrm{Ru}_{2} \mathrm{Si}_{2}$ single crystal have been studied for the first time.

Single crystals of the $\mathrm{U}_{1-x} \mathrm{Ce}_{x} \mathrm{Ru}_{2} \mathrm{Si}_{2}$ system $(x=0.00,0.025,0.05,0.075)$ were grown from the melt by the Czochralski method in a modified tri-arc furnace [4]. The temperature dependence of magnetic susceptibility was measured up to room temperature in an applied magnetic field with induction $\mu_{0} H=1 \mathrm{~T}$ using a commercial SQUID magnetometer.

The temperature dependence of magnetic susceptibility is shown in Fig. 1 The susceptibility measured perpendicular to the $c$-axis is almost independent of temperature. Along the $c$-axis it increases with decreasing temperature, reaches a broad maximum below $60 \mathrm{~K}$ and falls down. The broad maximum is connected with a transition to the coherent Kondo state. With Ce-alloying the absolute value of the broad maximum increases and the temperature of the maximum $T_{\max }$ shifts to lower temperature $\left(T_{\max }=57 \mathrm{~K}, 50 \mathrm{~K}, 43 \mathrm{~K}\right.$ and $38 \mathrm{~K}$ for $x=0,0.025,0.05$ and 0.075 , respectively).

The temperature dependence of the susceptibility follows the Curie-Weiss law at high temperatures. The set of parameters obtained from the Curie-Weiss law differs in different temperature ranges. In the high temperature range (300$140 \mathrm{~K}$ ) we obtained $\mu_{\mathrm{eff}}=3.64 \mu_{\mathrm{B}}, 3.77 \mu_{\mathrm{B}}, 3.87 \mu_{\mathrm{B}}$ and $|\Theta|=112 \mathrm{~K}, 107 \mathrm{~K}, 105 \mathrm{~K}$ for $x=0,0.05,0.075$, respectively. In the low temperature range $(140-80 \mathrm{~K})$ we obtained $\mu_{\text {eff }}=3.95 \mu_{\mathrm{B}}, 4.07 \mu_{\mathrm{B}}, 4.13 \mu_{\mathrm{B}}$ and $|\Theta|=167 \mathrm{~K}, 148 \mathrm{~K}, 138 \mathrm{~K}$ for $x=0$, $0.05,0.075$, respectively.

Our results of susceptibility measurements in the paramagnetic region with the magnetic field applied along the $c$-axis suggest that in $\mathrm{U}_{1-x} \mathrm{Ce}_{x} \mathrm{Ru}_{2} \mathrm{Si}_{2}$ with a higher concentration of $\mathrm{Ce}$, the $f$-electrons behave more localised (decrease in $T_{\max }$ and increase in $\mu_{\text {eff }}$ ). This result correlates with a more narrow width of the $4 f$-band of $\mathrm{Ce}$ and with an increase in interatomic distances with Ce-alloying [5]. The antiferromagnetic correlation increases with decreasing temperature $(|\Theta|$ increases). This phenomenon is usually interpreted as due to a decrease in Kondo temperature resulting from the depopulation of crystal field levels. The Ce-alloying reduces the antiferromagnetic correlation $(|\Theta|$ decreases). The results correspond with our previous results [6].

The insets of Fig. 1a and Fig. 1b show an anomaly in susceptibility data below $20 \mathrm{~K}$ for $x=0,0.025$ and 0.05 . The onset of the anomaly we connect with an antiferromagnetic transition. We did not find any anomaly in susceptibility data for $x=0.075$. The Néel temperature was estimated to be $T_{\mathrm{N}}=17.9 \mathrm{~K}, 15 \mathrm{~K}$ and $12 \mathrm{~K}$ for $x=0,0.025$ and 0.05 , respectively. The results are in good agreement with susceptibility measurements on $\mathrm{U}_{0.99} \mathrm{Ce}_{0.01} \mathrm{Ru}_{2} \mathrm{Si}_{2}$ and heat capacity mea- 

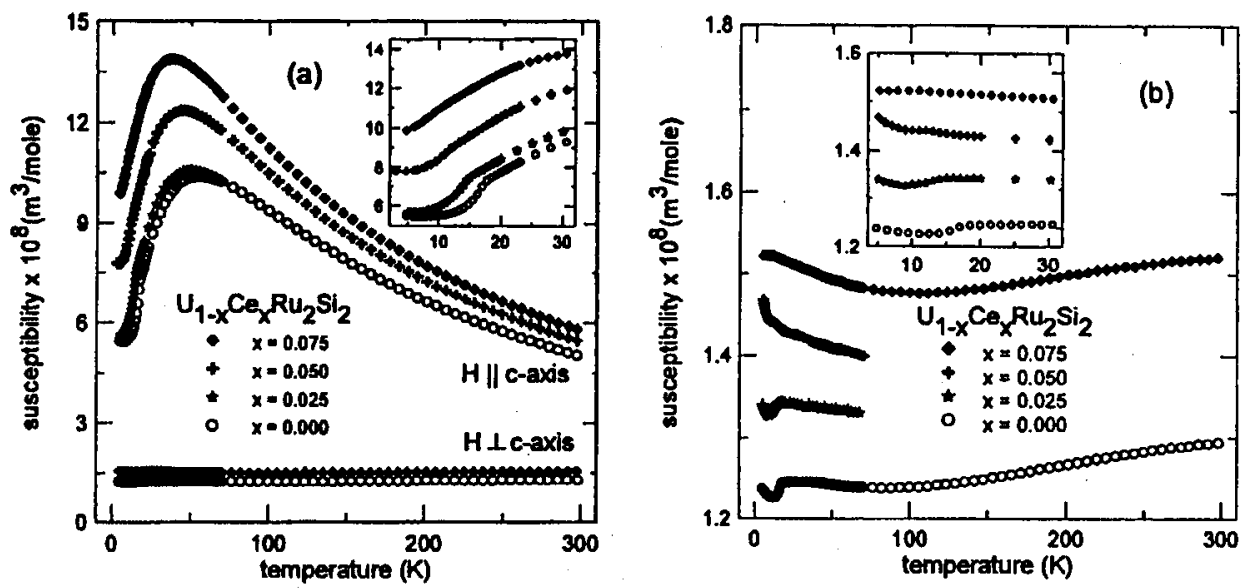

Fig. 1. Temperature dependence of magnetic susceptibility of $\mathrm{U}_{1-x} \mathrm{Ce}_{x} \mathrm{Ru}_{2} \mathrm{Si}_{2}$ for $x \leq 0.075$. (a) The susceptibility measured along and perpendicular to the $c$-axis. The inset shows susceptibility for $H \| c$-axis in the vicinity of $T_{\mathrm{N}}$. (b) Magnetic susceptibility for the magnetic field applied perpendicular to the $c$-axis. The inset shows magnetic susceptibility in the vicinity of $T_{N}$.
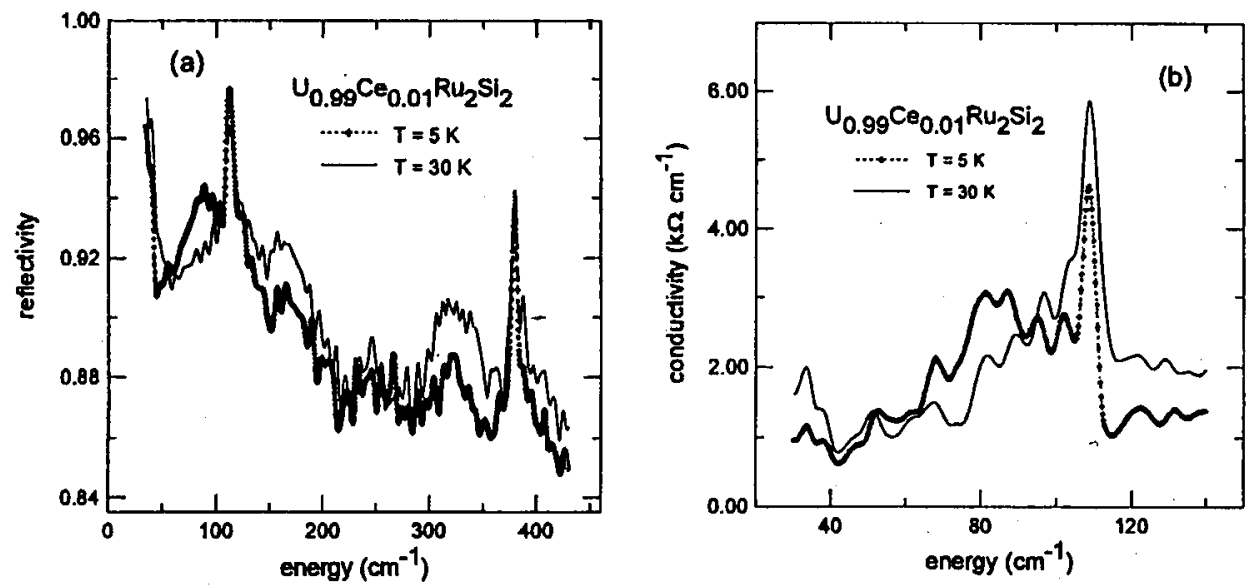

Fig. 2. Optical data of $\mathrm{U}_{0.99} \mathrm{Ce}_{0.01} \mathrm{Ru}_{2} \mathrm{Si}_{2}$ in the coherent state above the Néel temperature $(T=30 \mathrm{~K})$ and below the Néel temperature $(T=5 \mathrm{~K})$. (a) Optical reflectivity. (b) Optical conductivity.

surements on $\mathrm{U}_{1-x} \mathrm{Ce}_{x} \mathrm{Ru}_{2} \mathrm{Si}_{2}(x=0.01,0.05)$ [6]. Alloying with Ce decreases the Néel temperature and destroys an antiferromagnetic order for $x>0.05$.

For our optical investigations we used a well-defined single crystal $\mathrm{U}_{0.99} \mathrm{Ce}_{0.01} \mathrm{Ru}_{2} \mathrm{Si}_{2}$ [4]. Reflectivity was measured with a Bruker IFS $113 \mathrm{v}$ spectrometer equipped with standard detectors for the range from 20 to $5000 \mathrm{~cm}^{-1}$ with a resolution of $1 \mathrm{~cm}^{-1}$. The measurements were performed at $5 \mathrm{~K}, 30 \mathrm{~K}$ and 
room temperature. Figure 2a shows the optical reflectivity of $\mathrm{U}_{0.99} \mathrm{Ce}_{0.01} \mathrm{Ru}_{2} \mathrm{Si}_{2}$ at two different temperatures: below the Neel temperature $(T=5 \mathrm{~K})$ and above the Néel temperature $(T=30 \mathrm{~K})$. The sharp features at $111 \mathrm{~cm}^{-1}$ and $379 \mathrm{~cm}^{-1}$ are due to phonons. The absorption minimum near $44 \mathrm{~cm}^{-1}$ and the feature between $44 \mathrm{~cm}^{-1}$ and $90 \mathrm{~cm}^{-1}$ seen in $5 \mathrm{~K}$ data are probably caused by an energy gap associated with the antiferromagnetic transition.

The optical conductivity shown in Fig. 2b is obtained through the KramersKronig transformation of the reflectivity spectra. Figure $2 b$ shows the conductivity below and above $T_{\mathrm{N}}$. The conductivity taken at $5 \mathrm{~K}$ is very low below $43 \mathrm{~cm}^{-1}$ and rises rapidly to a peak near $80 \mathrm{~cm}^{-1}$. The shape of this feature is very similar to the peak which has been seen in the optical conductivity. of $\mathrm{URu}_{2} \mathrm{Si}_{2}$ in the spin-density-wave (SDW) state [7]. The energy of the gap $\left(44 \mathrm{~cm}^{-1}\right)$ has got nearly the same value as for $\mathrm{URu}_{2} \mathrm{Si}_{2}$. Our optical investigations support the assumption that the origin of magnetic ordering in $\mathrm{U}_{0.99} \mathrm{Ce}_{0.01} \mathrm{Ru}_{2} \mathrm{Si}_{2}$ is the same as in heavy-fermion superconductor $\mathrm{URu}_{2} \mathrm{Si}_{2}$ and can be interpreted as being due to a SDW gap formation in the vicinity of the Fermi level.

\section{References}

[1] C. Broholm, J.K. Kjems, W.J.L. Buyers, P. Matthews, T.T.M. Palstra, A.A. Menovsky, J.A. Mydosh, Phys. Rev. Lett. 58, 1467 (1987).

[2] M.B. Maple, J.W. Chan, Y. Dalichaouch, T. Kohara, C. Rossel, M.S. Torikach vili, M.W. Elfresh, J.D. Thomson, Phys. Rev. Lett. 56, 185 (1986).

[3] T.T.M. Palstra, A.A. Menovsky, J.A. Mydosh, Phys. Rev. B 33, 6527 (1986).

[4] M. Mihalik, N.H. Kim-Ngan, F.E. Kayzel, A.A. Menovsky, R.W.A. Hendrix, T.J. Gortenmulder, J. Cryst. Growth 134, 342 (1993).

[5] S. Maťaš, M. Mihalik, V. Kavečanký, J. Kováč, A.A. Menovsky, Acta Phys. Slovaca 46, 197 (1996).

[6] M. Mihalik, S. Maťaš, K. Prokeš, H. Nakotte, N.H. Kim-Ngan, A.A. Menovsky, IEEE Trans. Magn. 30, 1142 (1994).

[7] D.A. Bonn, J.G. Garret, T. Timusk, Phys. Rev. Lett. 61, 1305 (1988). 